Original Research

\title{
Addiction to Social Networks and Online Games: A Wrists' Pain Survey in Students
}

\section{Mohammad Babamiri' ${ }^{1}$ Rashid Heidari Moghaddam ${ }^{2}$, Fakhroddin Ghasemi ${ }^{3}$, Mohammad Ghaffari ${ }^{4}$, Mahdi Raziee ${ }^{5}$, Khadijeh Bandeh Elahi ${ }^{6}$, Alireza Mortezapour ${ }^{7 *}$}

1. Social Determinants of Health Research Center, Department of Ergonomics, School of public Health, Hamadan University of Medical Sciences, Hamadan, Iran

2. Health Sciences Research Center, Department of Ergonomics, School of Public Health, Hamadan University of Medical Sciences, Hamadan, Iran

3. Occupational Health and Safety Research Center, Department of Ergonomics, School of Public Health, Hamadan University of Medical Sciences, Hamadan, Iran

4. PhD Student of Biostatistics, School of Public Health, Hamadan University of Medical Sciences, Hamadan, Iran

5. MSc Student of Ergonomics, School of Public Health, Hamadan University of Medical Sciences, Hamadan, Iran

6. MSc Student of Health Education and Health Promotion, School of Public Health, Hamadan University of Medical Sciences, Hamadan, Iran

7. PhD Student of Ergonomics, School of Public Health, Hamadan University of Medical Sciences, Hamadan, Iran

\begin{tabular}{ll}
\hline \multicolumn{2}{c}{ Article Info } \\
\hline Original Article \\
Received: & $2018 / 09 / 20$ \\
Accepted: & $2018 / 11 / 29$ \\
Published Online: & $2018 / 12 / 26$
\end{tabular}

DOI: $10.30699 /$ jergon.6.3.4

Use your device to scan and read the article online

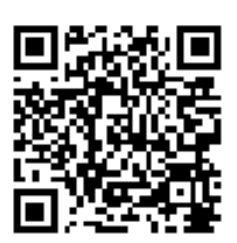

Corresponding Information

Alireza Mortezapour

PhD Student of Ergonomics, School of Public Health, Hamadan University of Medical Sciences, Hamadan, Iran Email:

amortezapour258@gmail.com

\begin{abstract}
Background and Objectives: Nowadays, the use of the Internet among students has become widespread. Addiction to virtual networks and online games can have various consequences, including the threat of musculoskeletal system in these people. The purpose of this study was to determine the effect of addiction to social networks and online games on students' wrist pain.
\end{abstract}

Methods: This study was conducted among 665 students. Data collection was done through three questionnaires of problematic use of online games, social networking addiction and Nordic musculoskeletal disorders. Data were compared by Chi-square and independent T-test and finally, logistic regression model was presented at a significant level of 0.05 .

Results: The results showed that social network and online game addiction can increase the risk of pain in the wrists. Sex had a significant impact in the effect of social network addiction on pain in the wrist. Other results of the present study are the not significant differences in the age and level of education of the participants in the prevalence of wrist pain.

Conclusion: It can be stated that there is a likelihood of a relationship between social network and online game addiction with wrist pain. Therefore, due to high prevalence of this addiction among students, appropriate planning should be taken to reduce complications and injuries, especially in the wrist area of them.

Keywords: Behavioral addiction, Cyber space, Internet Game, Carpal Tunnel Syndromes, Wrist injury, Social media

Copyright (C) 2018, Journal of Ergonomics. This is an open-access article distributed under the terms of the Creative Commons Attribution-noncommercial 4.0 International License which permits copy and redistribute the material just in noncommercial usages, provided the original work is properly cited

How to Cite This Article:

Babamiri M, Haidari Moghaddam R, Ghasemi F, Ghaffari M, Razee M, Bandeh Ellahi K, et al .Addiction to Social Networks and Online Games: A Wrists' Pain Survey in Students. J Ergon. 2018; 6 (3): 33-42 


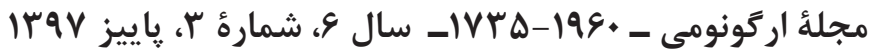

\author{
مقاله يزوهشى
}

\section{اعتياد به شبكههاى اجتماعى و بازىهاى آنلاين: بررسى درد در ناحية مج دست در دانشجويان}

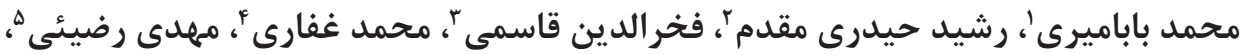

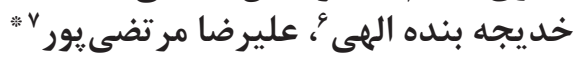

|. استاديار، مركز تحقيقات عوامل اجتماعى مؤثر بر سلامت، كروه اركونومى، دانشكدة بهداشت، دانشكاه علوم يزشكى همدان،

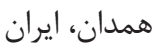

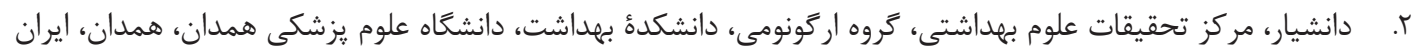

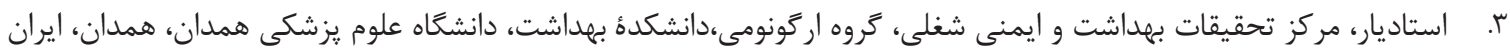

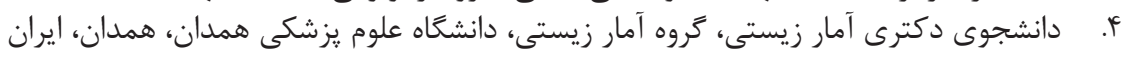

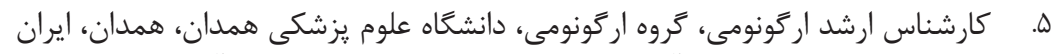

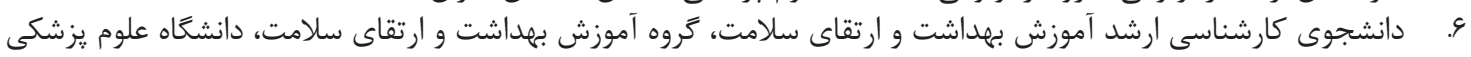

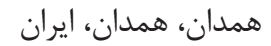

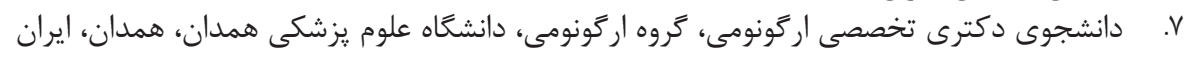

\begin{tabular}{|c|c|}
\hline جكيده & اطلاعات مقاله \\
\hline 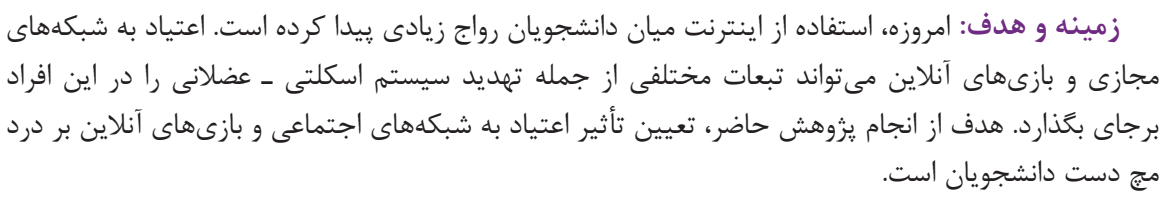 & 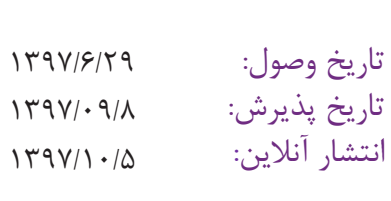 \\
\hline 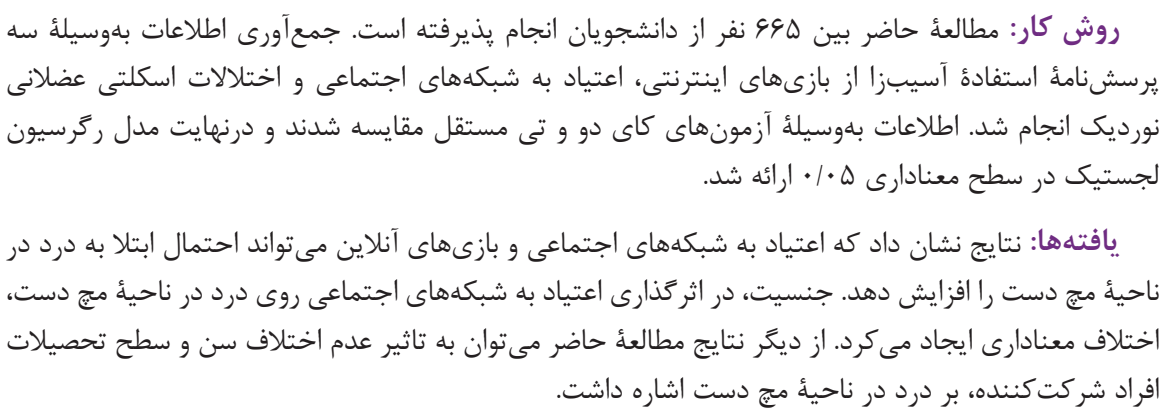 & 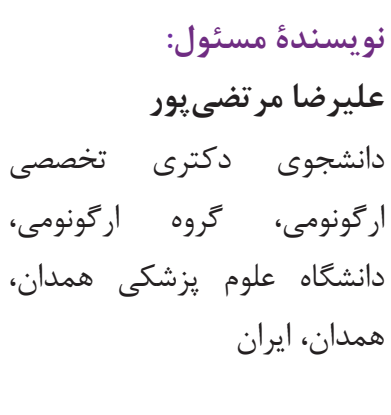 \\
\hline 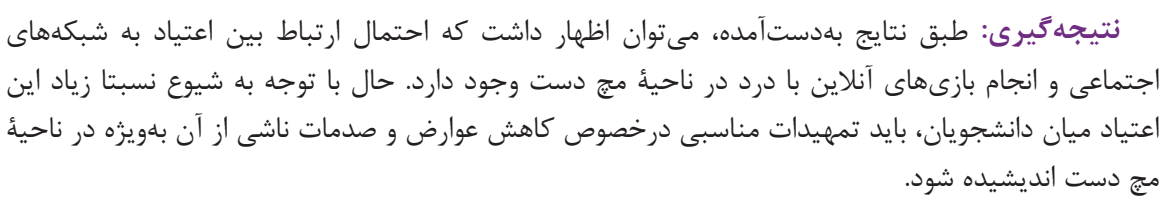 & يست الكترونيك: \\
\hline شبكؤ اجتماعى وازى كليدى: اعتياد رفتارى، فضاى مجازى، بازى اينترنتى، نشانكان مجراى كاريال، آسيب مج دست، & \\
\hline
\end{tabular}

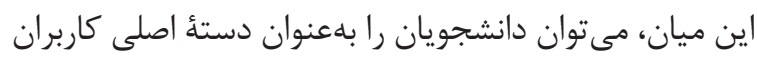
مقدمه

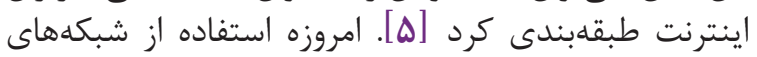

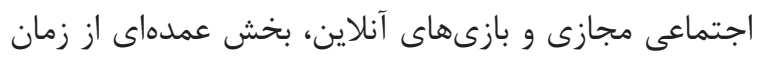

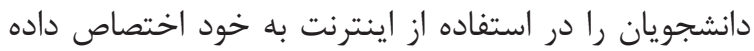

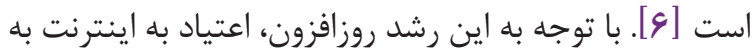

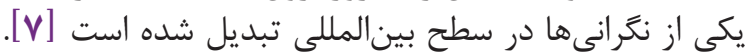

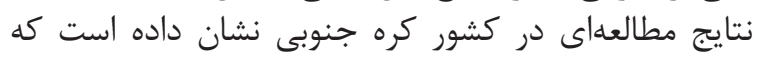

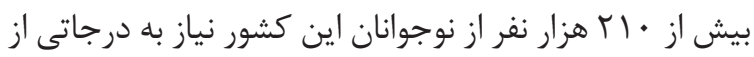

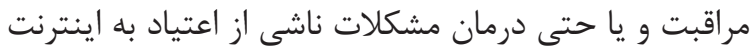

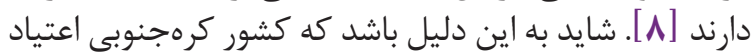

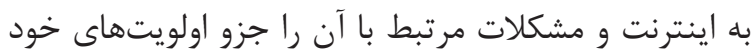

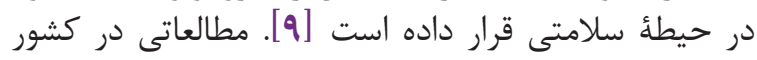

امروزه، استفاده از اينترنت بهعنوان منبع اصلى اطلاعات

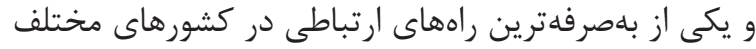

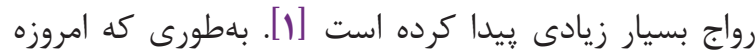

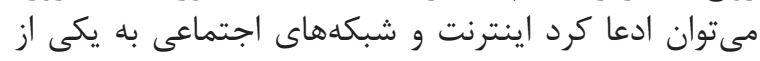

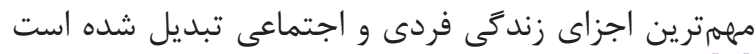

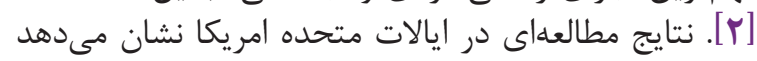

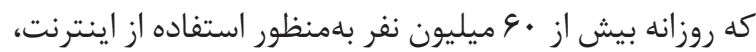

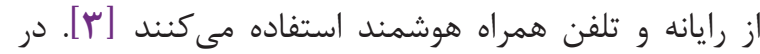

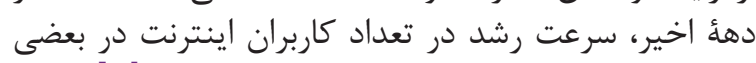

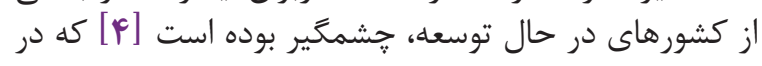




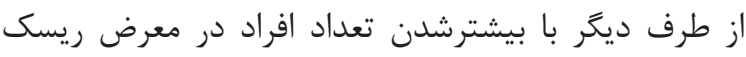

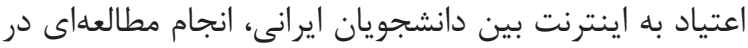

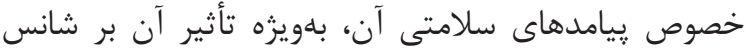

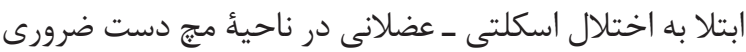

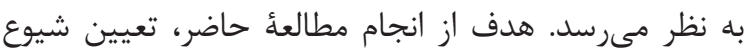

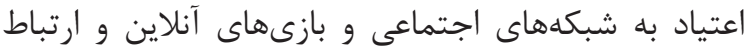

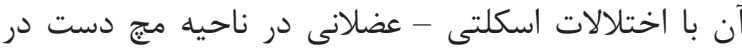
دانشجويان دانشگاه علوم يزشكى همدان است استى

\section{روش كار}

مطالعة حاضر بلهورت مقطعى طرحريزى و اجرا شده

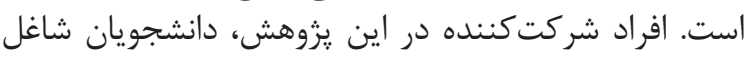

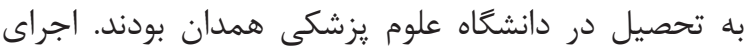

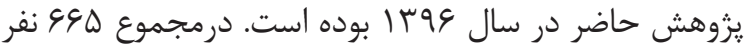

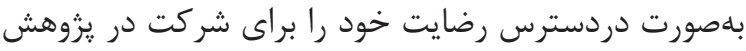

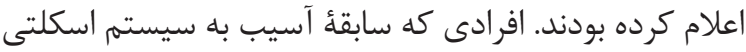

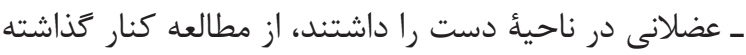

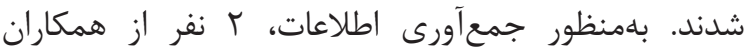

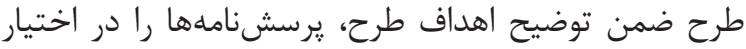
شركت كنند

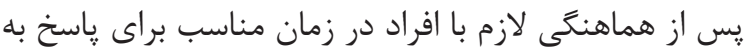

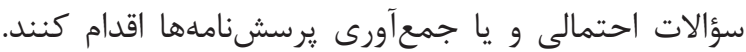

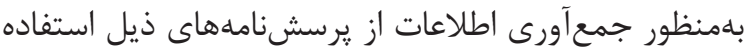

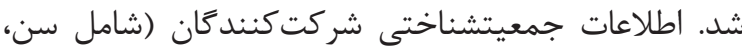

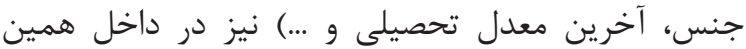

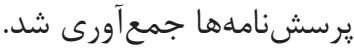

\section{يرسشنامةٔ اعتياد به شبكههاى اجتماعى مجازى}

اين : يرسشنامه داراى سأ گويه بلهورت ليكرت لهتايى

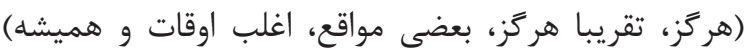

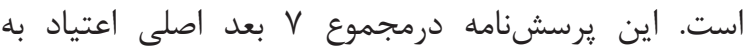

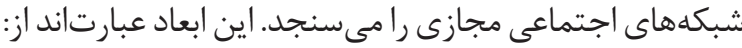

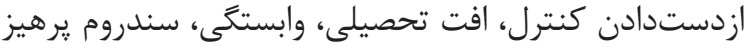

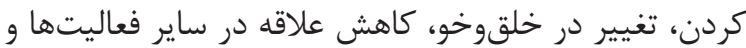

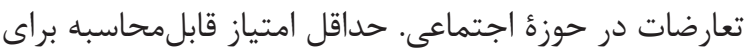

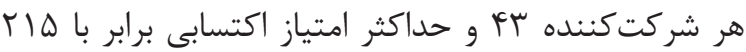

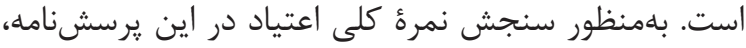

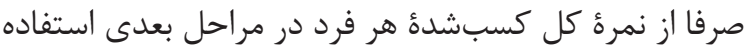

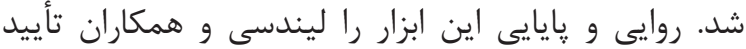

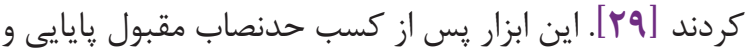

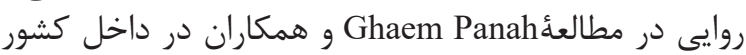
نيز استفاده شده است [." رب].

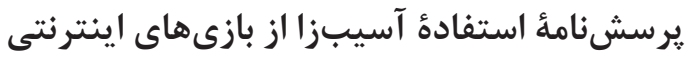

براى جمعآورى اين شاخص، از ابزار بازى مشكلساز

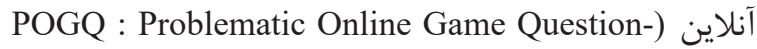

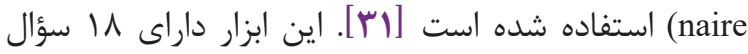
است كه شركت كنند

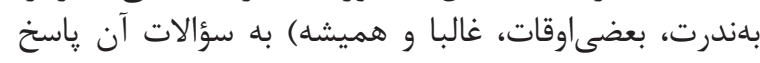

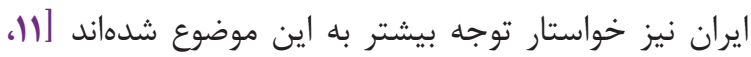

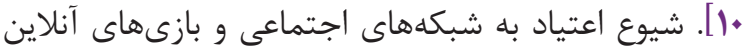

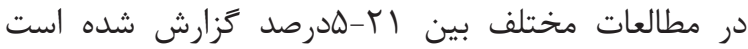

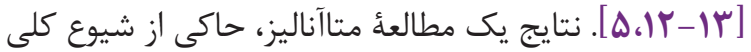

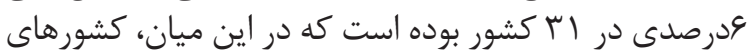

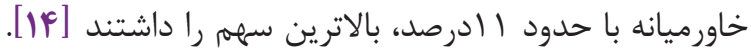

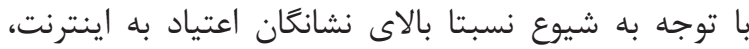

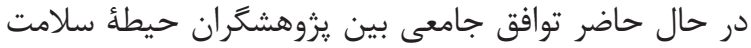

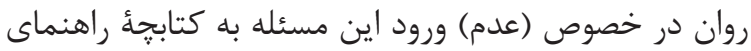

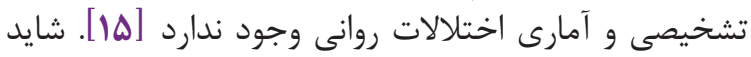

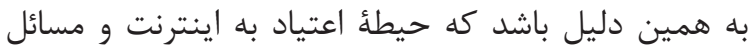

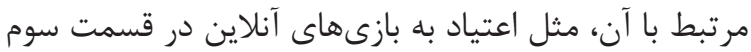

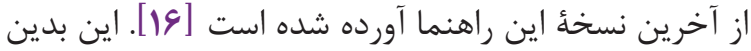

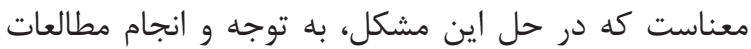

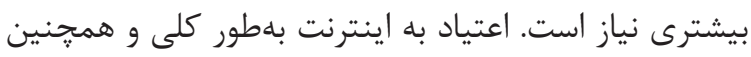

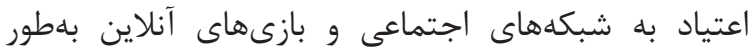

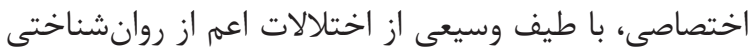

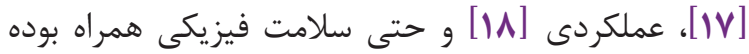

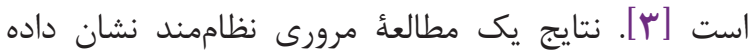

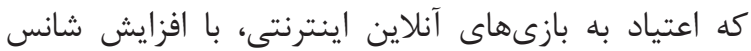

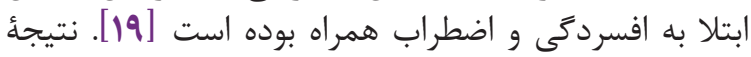

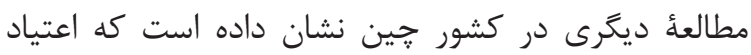

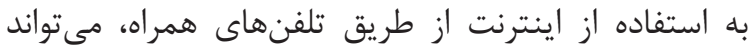

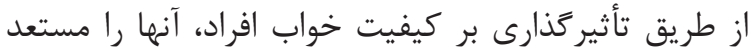

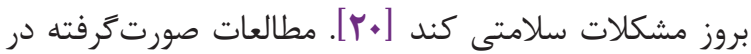

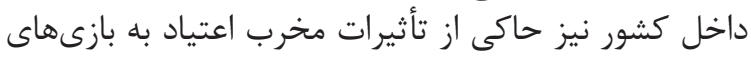

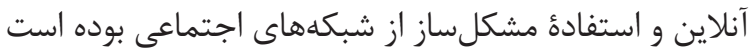

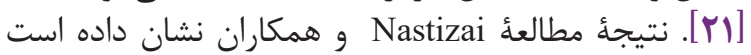

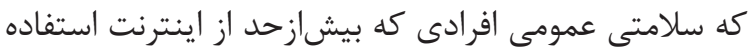

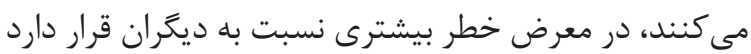

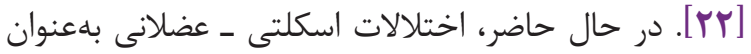

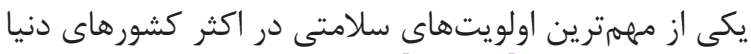

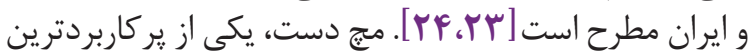

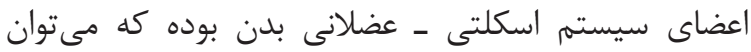

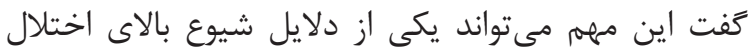

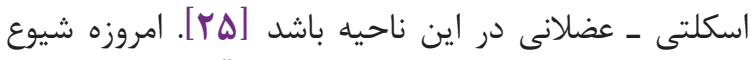

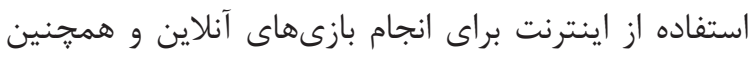

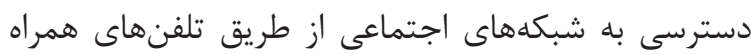

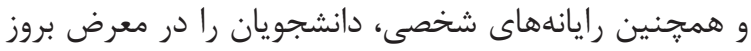

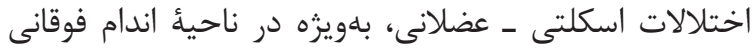

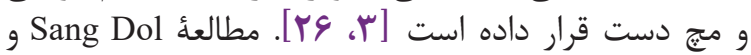

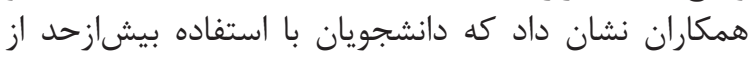

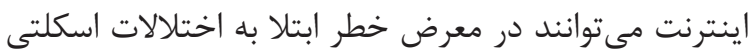

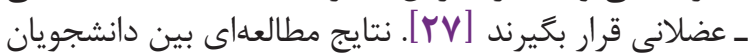

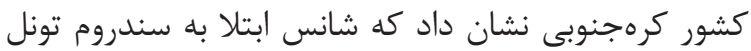

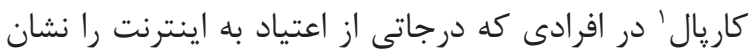

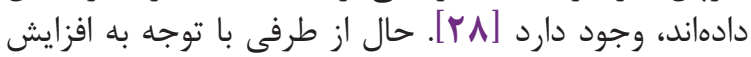

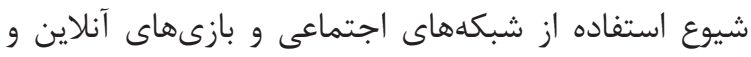




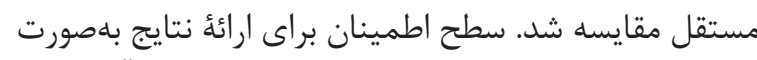

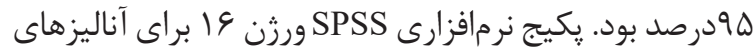

اشارهشده، استفاده شد.

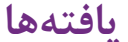

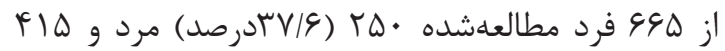

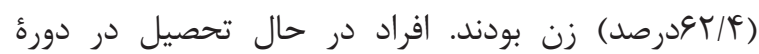

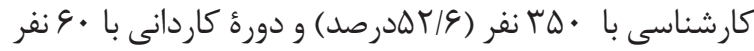

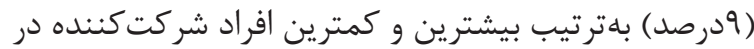

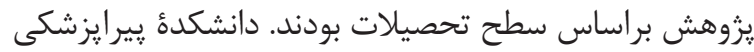

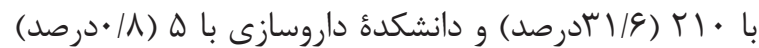

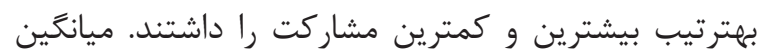

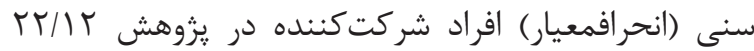

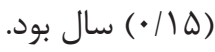

جدول شماره | فراوانى متغيرهاى جنسيت، سطح

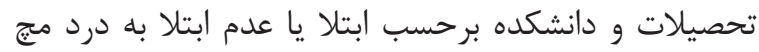

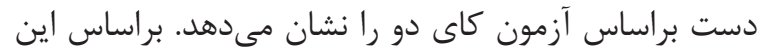

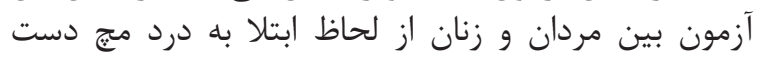

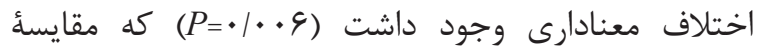

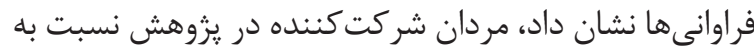

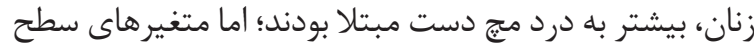

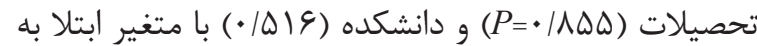
مجز دست ارتباط معنادارى نداشتند.

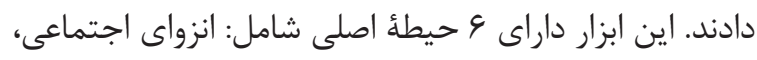

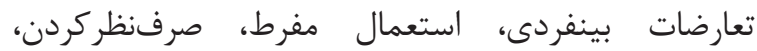

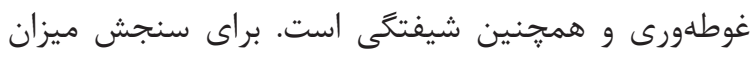

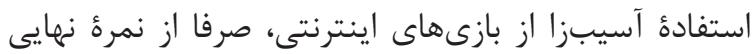

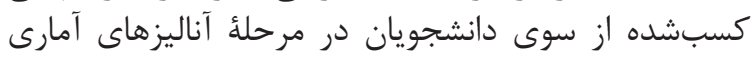

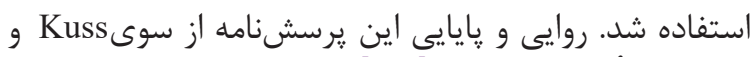

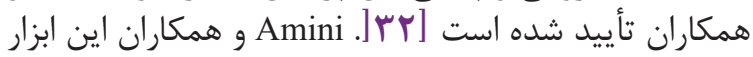

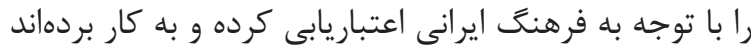

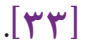

\section{يرسشنامةٔ اختلالات اسكلتى ـ عضلانى (در ناحية مج هر دو دست)}

اطلاعات اختلالات اسكلتى ـ عضلانى دانشجويان در ناحئ

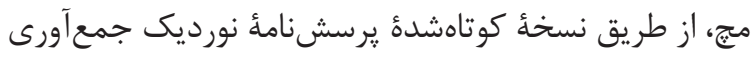

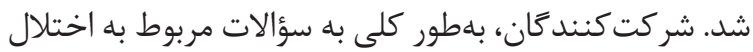

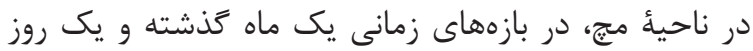

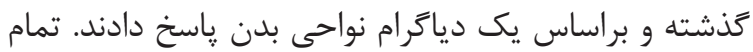

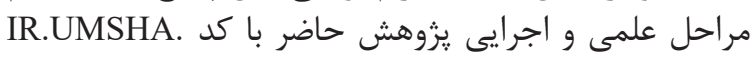

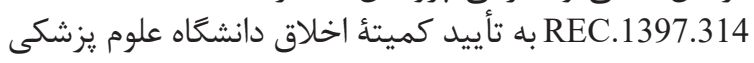
همدان رسيده است.

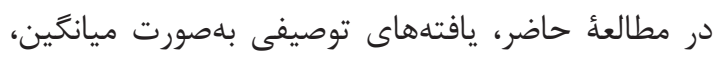

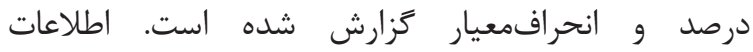

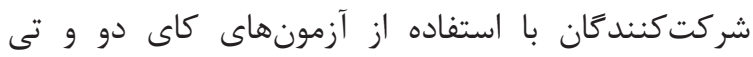

جدول شماره ا. فراوانى متغيرهاى جنسيت و سطح تحصيلات براساس افراد سالم و مبتلا به مجدرد

\begin{tabular}{|c|c|c|c|c|c|}
\hline \multirow{2}{*}{ معنادارى } & \multirow{2}{*}{ آماره آزمون } & \multicolumn{2}{|c|}{ درد مجز دست } & \multirow[b]{2}{*}{ رده } & \multirow[b]{2}{*}{ متغير } \\
\hline & & خير (درصد) & بله (درصد) & & \\
\hline \multirow[t]{2}{*}{$.1 \cdot .9$} & $V / 99$ & $|\Delta|(\xi \cdot / f)$ & $99(\% 9 / 9)$ & مرد & \multirow{2}{*}{ جنسيت } \\
\hline & & $r q Y(V \cdot / \Lambda)$ & $|r|(r q / r)$ & زن & \\
\hline \multirow[t]{2}{*}{$\cdot / \wedge \Delta \Delta$} & $\cdot 1 \cdot r$ & ऍ & $T Y(Y G / V)$ & كاردانى & \multirow{2}{*}{ سطح تحصيلات } \\
\hline & & $r$ G ( $(Q V / F)$ & 114 (rr/G) & كارشناسى & \\
\hline
\end{tabular}

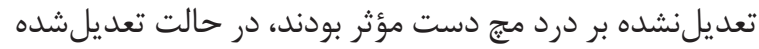

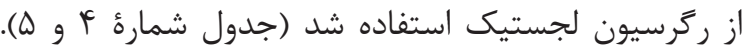

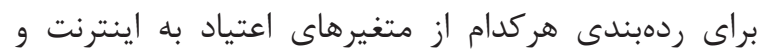

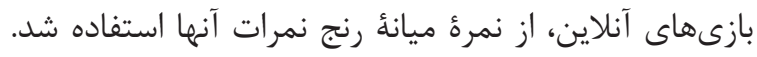

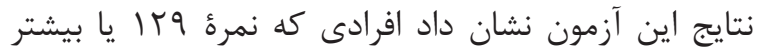

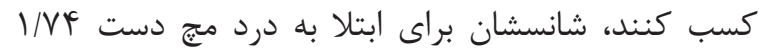

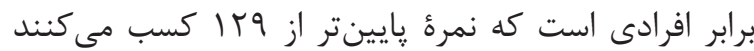

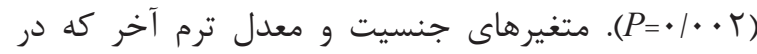

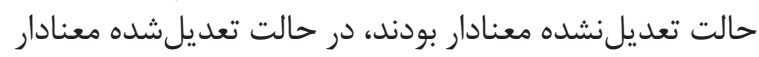

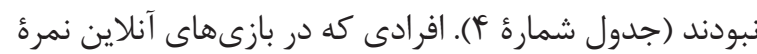

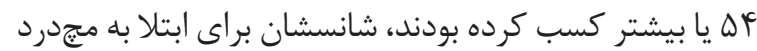

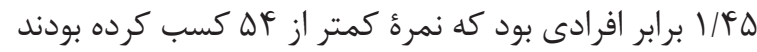

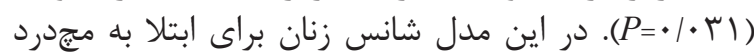

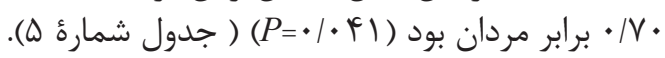

جدول شماره ז، ميانگين سنى و معدل آخرين ترم افراد

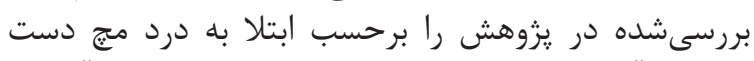

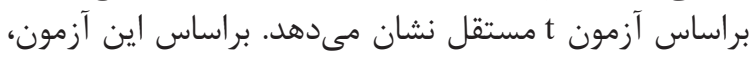

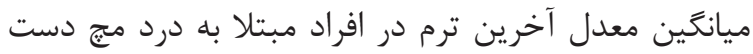

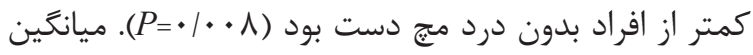

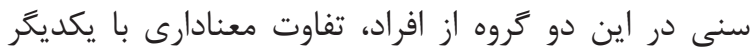

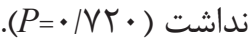
براى بررسى تأثير اعتياد به اينترنت و بازىهاى آنلاين بر

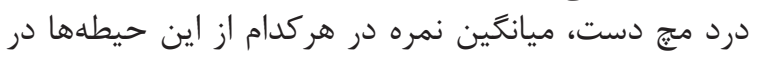

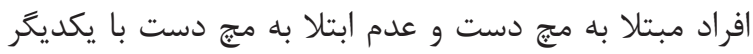

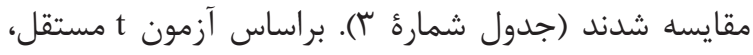

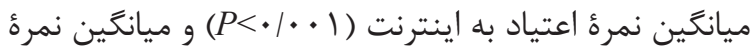

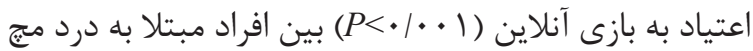
دست بيشتر از افراد سالم بود. براى بررسى تأثير هركدام از متغيرهايى كه در حالت 


$$
\text { جدول شماره r. ميانكين سنى و معدل آخرين ترم افراد بررسى مبده در يخوهش را براساس }
$$

\begin{tabular}{|c|c|c|c|c|c|}
\hline معنادارى & آماره آزمون & ميانگين (انحراف استاندارد) & تعداد & درد مج دست & 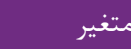 \\
\hline \multirow[t]{2}{*}{. IVT. } & 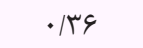 & $r T / 19(\cdot / 19)$ & FFa & خير & \multirow{2}{*}{ سن } \\
\hline & & $T Y / \cdot \Delta(\cdot / T Y)$ & rT. & بله & \\
\hline \multirow[t]{2}{*}{$\cdot / \cdot \wedge$} & $r / 9 V$ & $19 / 19(\cdot / \cdot v)$ & Fis & خير & \multirow{2}{*}{ آخرين ترم } \\
\hline & & IQ/Ar $(\cdot / 1 \cdot)$ & rt. & بله & \\
\hline
\end{tabular}

\begin{tabular}{|c|c|c|c|c|c|}
\hline 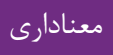 & آماره آزمون & ميانگين (انحراف استاندارد) & 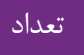 & درد مجز دست & 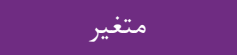 \\
\hline \multirow[t]{2}{*}{$<\cdot \mid \cdot \cdot 1$} & $\varphi / \mu$. & $\| N / T \&(1 / r K)$ & 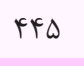 & خير & \multirow{2}{*}{ اعتياد به شبكههاى اجتماعى } \\
\hline & & ITN/Tr (I/9T) & $r t$. & بله & \\
\hline \multirow[t]{2}{*}{$<\cdot / \cdot \cdot 1$} & $\Gamma / \Lambda)$ & $\forall \varphi / \mid F(\cdot / V \cdot)$ & Fis & خير & \multirow{2}{*}{ اعتياد به بازى آنلاين } \\
\hline & & $\Delta \cdot \mid A r(1 / \cdot r)$ & tr. & بله & \\
\hline
\end{tabular}

جدول سّ. ميانگين نمرهٔ اعتياد به شبكههاى اجتماعى و بازىهاى آنلاين براساس افراد سالم و مبتلا به مجدرد

$$
\text { جدول شمارهُ F. بررسى اثر اعتياد به شبكههاى اجتماعى با تعديل اثر جنسيت و معدل آخرين ترم }
$$

\begin{tabular}{|c|c|c|c|}
\hline$\cdot / \cdot V T$ & 1 & مرد مرد & \multirow{2}{*}{ جنسيت } \\
\hline & $\cdot / V r(\cdot|\Delta|-1 / \cdot r)$ & زن & \\
\hline \multirow[t]{2}{*}{$\cdot \cdots r$} & 1 & $<1 r q$ & \multirow{2}{*}{ اعتياد به شبكههاى اجتماعى } \\
\hline & $1 / V F(I / T F-Y / F \Delta a)$ & $>=1149$ & \\
\hline - ITFF & / १९ $(\cdot \mid \Lambda \Gamma-1 / \cdot \Delta)$ & & معدل آخرينترم \\
\hline
\end{tabular}

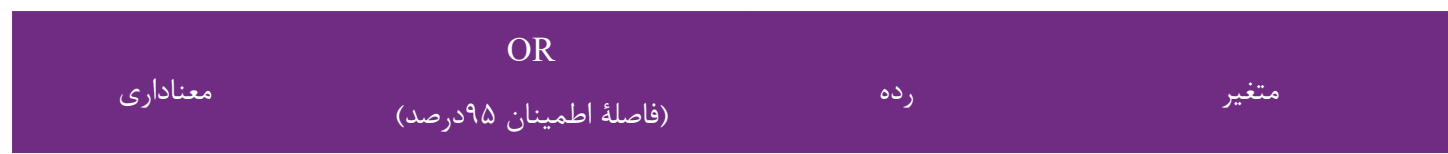

جدول شماره ه. بر رسى اثر اعتياد به بازى آنلاين با تعديل اثر جنسيت و معدل آخرين ترم

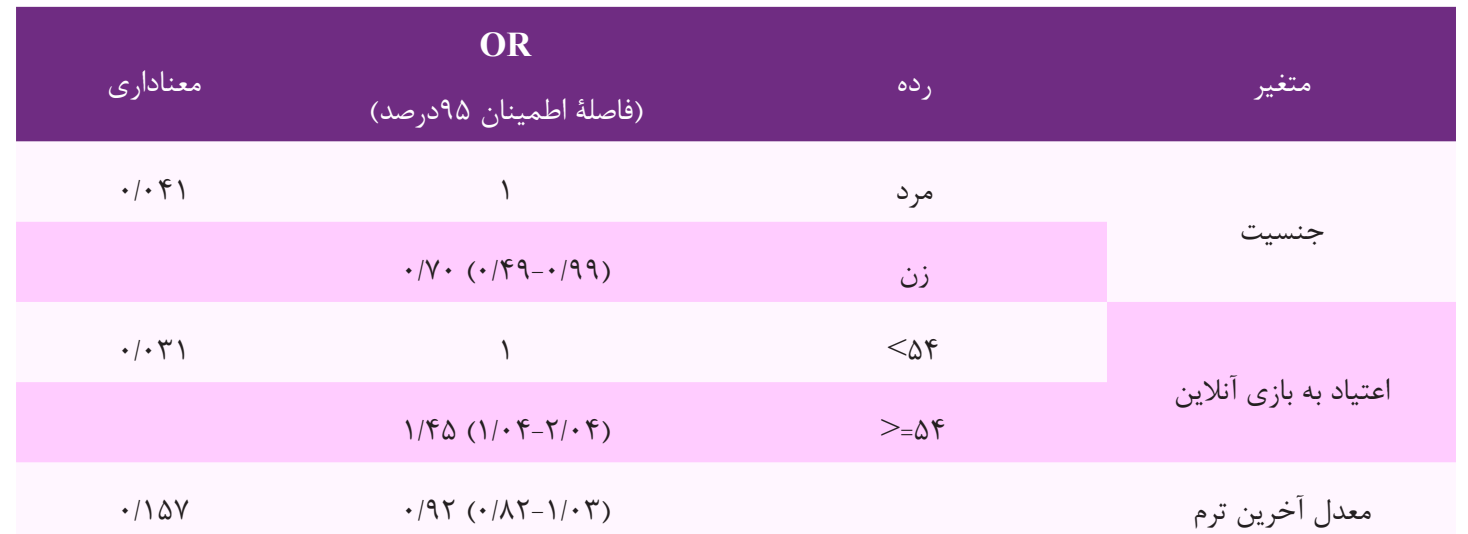




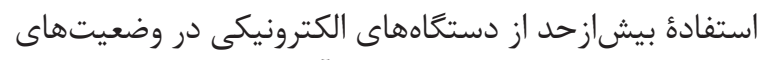

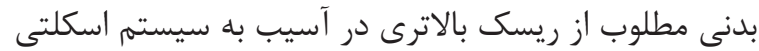

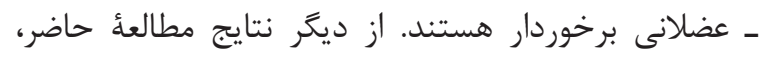

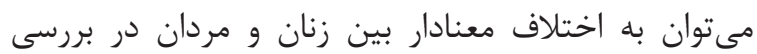

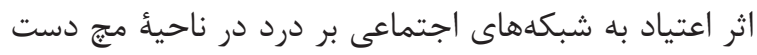

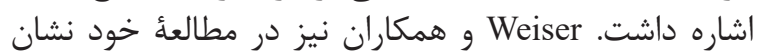

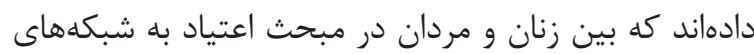

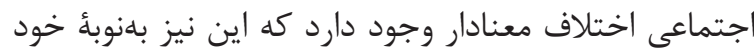

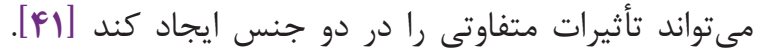

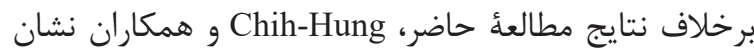

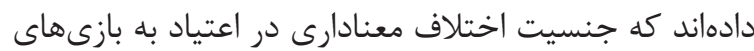

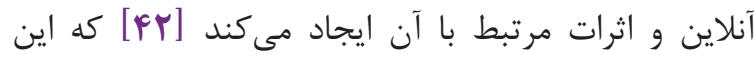

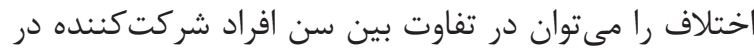

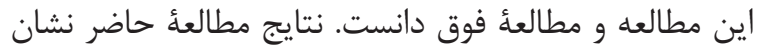

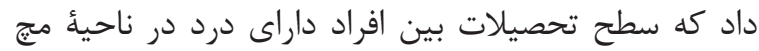

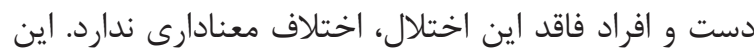

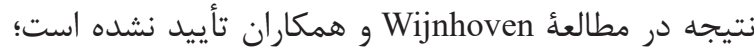

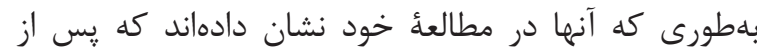

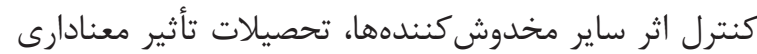

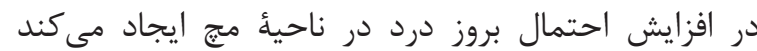

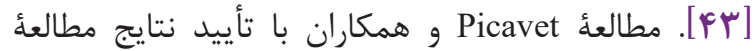

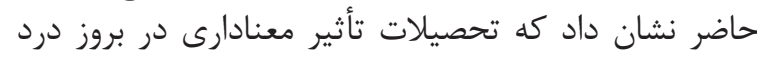

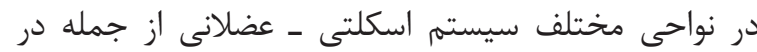

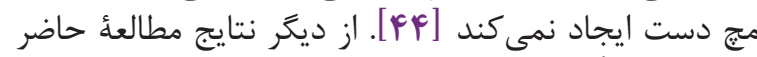

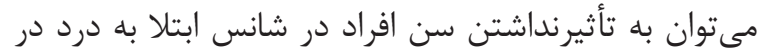

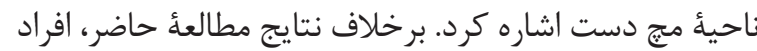

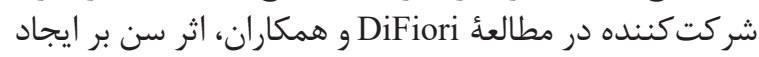

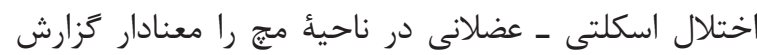

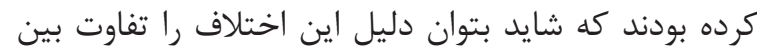

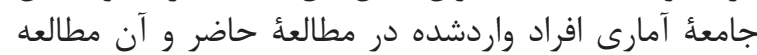

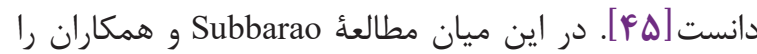

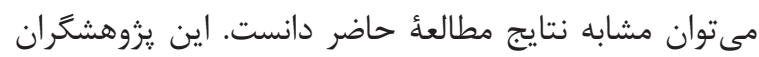

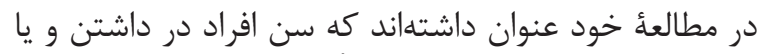

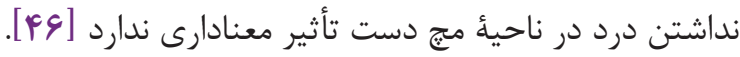

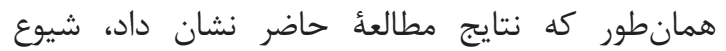

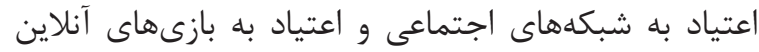

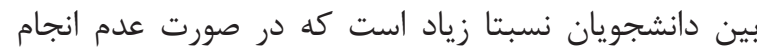

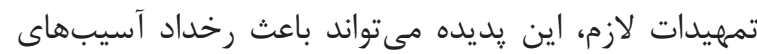

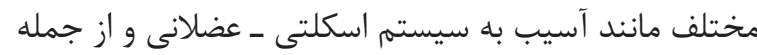

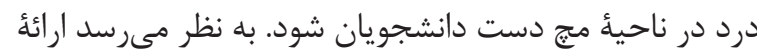

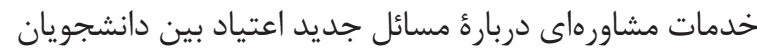

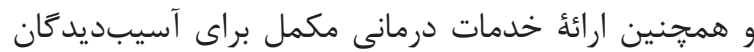

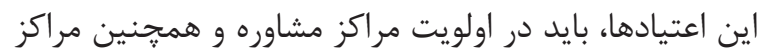
درمانى درون و بيرون از دانشخاهها قرار گيرداد

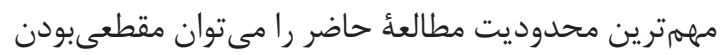

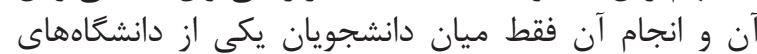

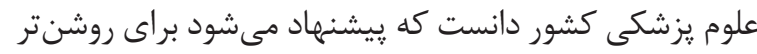

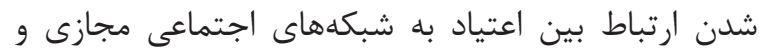
بازىهاى آنلاين از انواع ديخرى ازين مطالعات ماندان اجنداعى انواع طولى

\section{بحث و نتيجه تيرى}

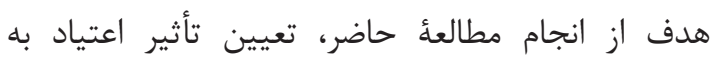

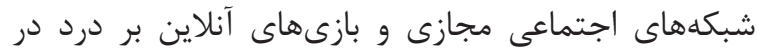

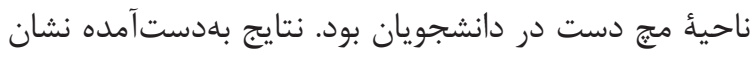

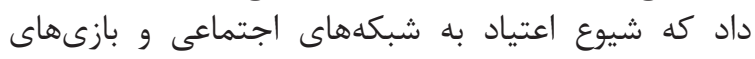

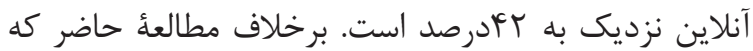

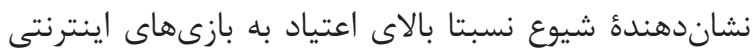

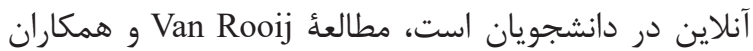

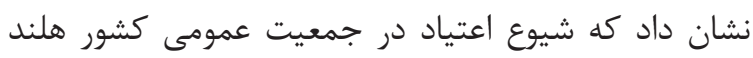

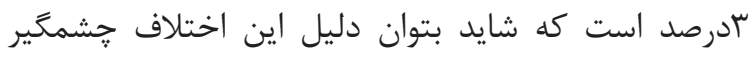

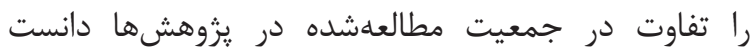

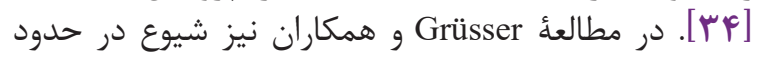

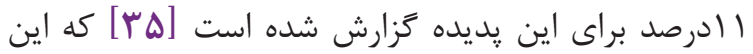

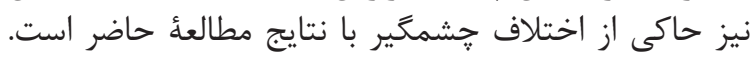

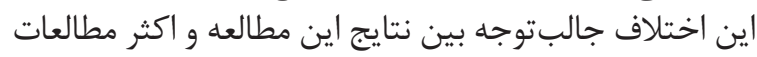

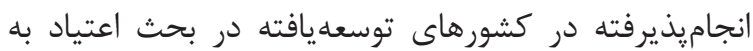

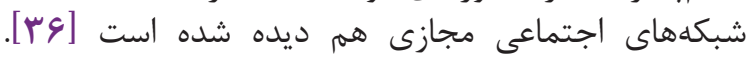

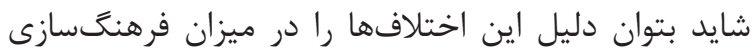

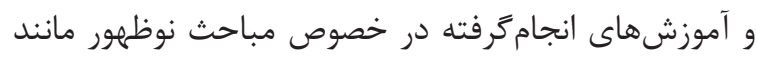

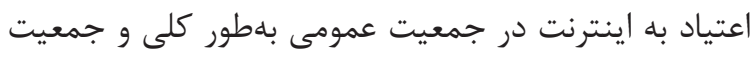

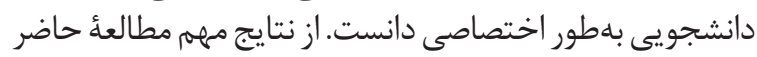

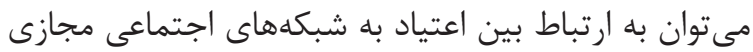

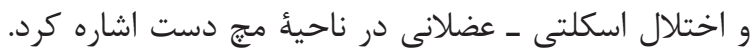

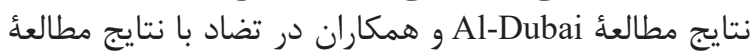

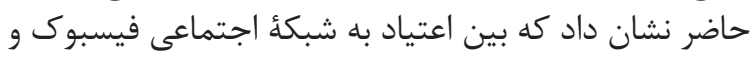

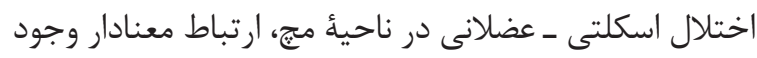

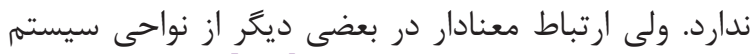

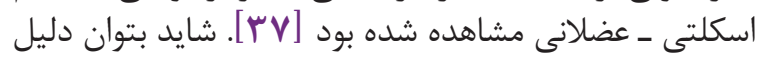

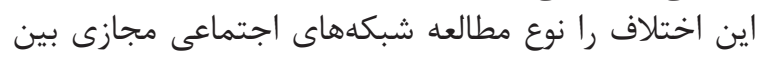

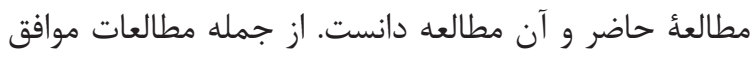

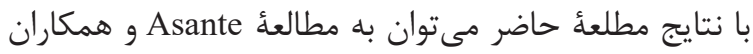

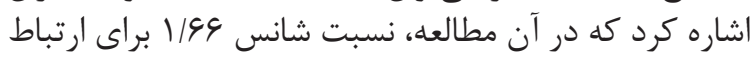

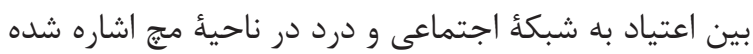

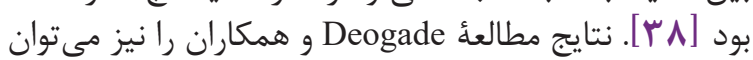

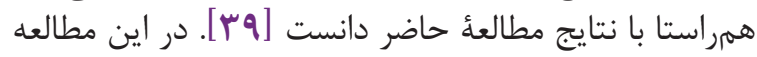

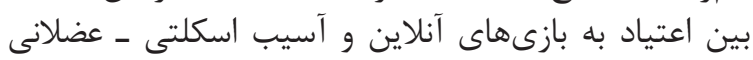

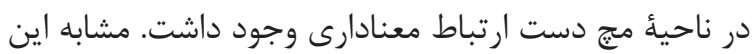

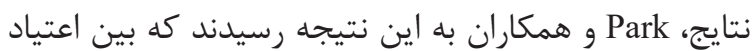

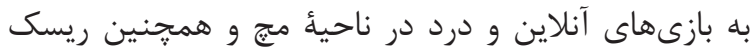

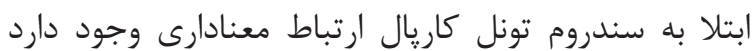

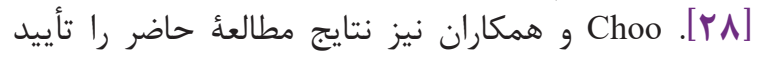

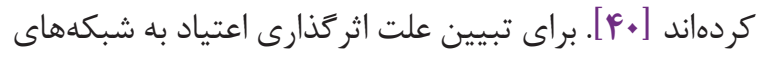

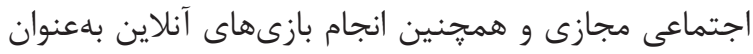

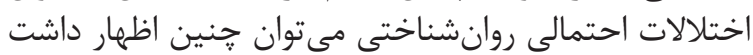

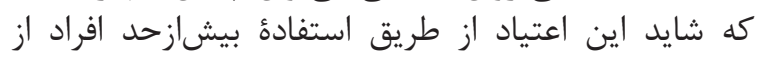

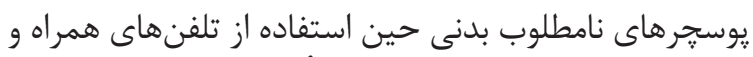

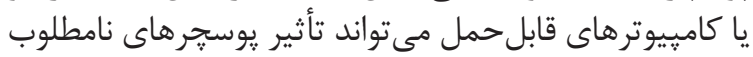

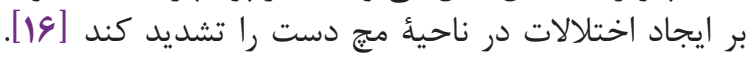

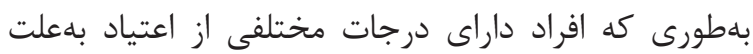


مستخرج از طرح تحقيقاتى مصوب معاونت يزوهشى دانشعاه

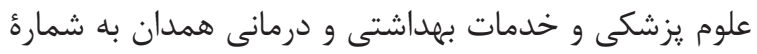

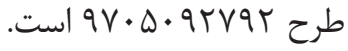
تعارض منافع

بين نويسندكان هيجَّونه تعارضى در منافع وجود ندارد.

\section{Reference}

1. Ozcinar Z. The relationship between internet addiction and communication, educational and physical problems of adolescents in North Cyprus. Journal of psychologists and counsellors in schools. 2011 Jun;21(1):22-32.

2. Durak HY. Modeling of variables related to problematic internet usage and problematic social media usage in adolescents. Current Psychology. 2018:1-3.

3. Clark DJ, Frith KH, Demi AS. The physical, behavioral, and psychosocial consequences of internet use in college students. CIN: Computers, Informatics, Nursing. 2004 May 1;22(3):153-61.

4. Ghamari F, Mohammadbeigi A, Mohammadsalehi N, Hashiani AA. Internet addiction and modeling its risk factors in medical students, Iran. Indian journal of psychological medicine. 2011 Jul;33(2):158. https://doi.org/10.4103/0253$\underline{7176.92068}$ PMID:22345841

5. Salehi M, Khalili MN, Hojjat SK, Salehi M, Danesh A. Prevalence of internet addiction and associated factors among medical students from Mashhad, Iran in 2013. Iranian Red Crescent Medical Journal. 2014 May;16(5). https://doi. org/10.5812/ircmj. 17256

6. Männikkö N, Billieux J, Kääriäinen M. Problematic digital gaming behavior and its relation to the psychological, social and physical health of Finnish adolescents and young adults. Journal of behavioral addictions. 2015 Dec 21;4(4):2818.https://doi.org/10.1556/2006.4.2015.040 PMID:26690623

7. Ryding FC, Kaye LK. "Internet Addiction": A conceptual minefield. International journal of mental health and addiction. 2018 Feb 1;16(1):225-32. https://doi.org/10.1007/s11469017-9811-6 PMid:29491771

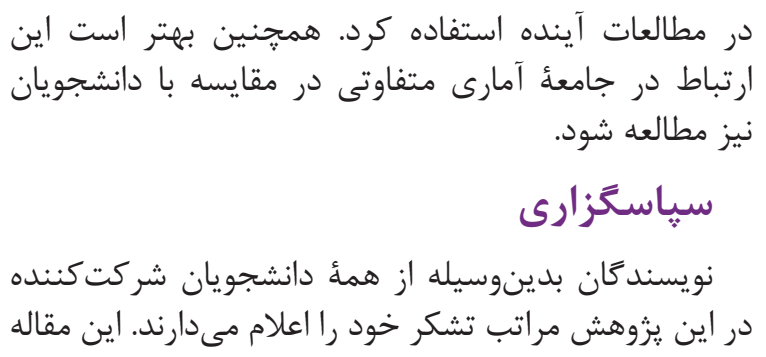

8. Choi YH. Advancement of IT and seriousness of youth Internet addiction. InInternational Symposium on the Counseling and Treatment of Youth Internet Addiction 2007 (p. 20). National Youth Commission Seoul, Korea.

9. Block JJ. Issues for DSM-V: Internet addiction. Am Psychiatric Assoc; 2008.

10. Kheirkhah F, Juibary AG, Gouran A. Internet addiction, prevalence and epidemiological features in Mazandaran Province, Northern Iran. Iranian Red Crescent Medical Journal. 2010 Mar $1 ; 12(2): 133$.

11. Ghassemzadeh L, Shahraray M, Moradi A. Prevalence of Internet addiction and comparison of Internet addicts and non-addicts in Iranian high schools. CyberPsychology \& Behavior. 2008 Dec 1;11(6):731-3. $\quad$ https://doi.org/10.1089/ cpb.2007.0243 PMID: $\underline{18954277}$

12. Mazhari S. The prevalence of problematic internet use and the related factors in medical students, Kerman, Iran. Addiction \& health. 2012;4(3-4):87.

13. Fu KW, Chan WS, Wong PW, Yip PS. Internet addiction: prevalence, discriminant validity and correlates among adolescents in Hong Kong. The British Journal of Psychiatry. 2010 Jun;196(6):486-92. https://doi.org/10.1192/bjp. bp.109.075002 PMID:20513862

14. Cheng C, Li AY. Internet addiction prevalence and quality of (real) life: A meta-analysis of 31 nations across seven world regions. Cyberpsychology, Behavior, and Social Networking. 2014 Dec 1;17(12):755-60. https://doi.org/10.1089/cyber.2014.0317 PMID: 25489876

15. Pies R. Should DSM-V designate "Internet addiction" a mental disorder?. Psychiatry (Edgmont). 2009 Feb;6(2):31.

16. Király O, Griffiths MD, Demetrovics Z. Internet 
gaming disorder and the DSM-5: Conceptualization, debates, and controversies. Curr Addict Rep. 2015:2(3):250-4. https://doi.org/10.1007/ s40429-015-0066-7

17. Young KS, Rogers RC. The relationship between depression and Internet addiction. Cyberpsychology \& behavior. 1998;1(1):25-8. https://doi. org/10.1089/cpb.1998.1.25

18. Kuss DJ, Griffiths MD, Binder JF. Internet addiction in students: Prevalence and risk factors. Computers in Human Behavior. 2013 May 1;29(3):959-66. https://doi.org/10.1016/j. chb.2012.12.024

19. Männikkö N, Ruotsalainen H, Miettunen J, Pontes HM, Kääriäinen M. Problematic gaming behaviour and health-related outcomes: A systematic review and meta-analysis. Journal of health psychology. 2017. https://doi. org/10.1177/1359105317740414

20. Xie X, Dong Y, Wang J. Sleep quality as a mediator of problematic smartphone use and clinical health symptoms. Journal of behavioral addictions. 2018 May 15:1-7. https://doi. org/10.1556/2006.7.2018.40

21. Alavi SS, Alaghemandan H, Maracy MR, Jannatifard F, Eslami M, Ferdosi M. Impact of addiction to internet on a number of psychiatric symptoms in students of isfahan universities, iran, 2010. International journal of preventive medicine. 2012 Feb;3(2):122.

22. Nastizai N. The relationship between general health and internet addiction. Zahedan Journal of Research in Medical Sciences. 2009 Apr $1 ; 11(1): 0-$

23. Tordrup D, Chouaid C, Cuijpers P, Dab W, van Dongen JM, Espin J, Jönsson B, Leonard C, McDaid D, McKee M, Miguel JP. Priorities for health economic methodological research: results of an expert consultation. International journal of technology assessment in health care. 2017;33(6):609-19. https://doi.org/10.1017/ $\underline{\mathrm{S} 0266462317000666}$

24. Moradi-Lakeh M, Forouzanfar MH, Vollset SE, El Bcheraoui C, Daoud F, Afshin A, Charara R, Khalil I, Higashi H, El Razek MM, Kiadaliri AA. Burden of musculoskeletal disorders in the Eastern Mediterranean Region, 1990-2013: findings from the Global Burden of Disease Study 2013.
Annals of the rheumatic diseases. 2017 Feb 16:annrheumdis-2016.

25. Malchaire JB, Cock NA, Robert AR. Prevalence of musculoskeletal disorders at the wrist as a function of angles, forces, repetitiveness and movement velocities. Scandinavian journal of work, environment \& health. 1996 Jun 1:176-81._ https://doi.org/10.5271/sjweh.128 PMID: $\underline{837262}$

26. Sharan D, Mohandoss M, Ranganathan R, Jose J. Musculoskeletal disorders of the upper extremities due to extensive usage of hand held devices. Annals of occupational and environmental medicine. 2014 Dec;26(1):22. https://doi.org/10.1186/ s40557-014-0022-3 PMID:25852936

27. Dol KS. Fatigue and pain related to internet usage among university students. Journal of physical therapy science. 2016;28(4):1233-7. https:// doi.org/10.1589/jpts.28.1233 PMID:27190458

28. Park SY, Lim WT, Kim YJ, Lee SW, Yi CH. The relationship between addiction to online games and carpal tunnel syndrome in college students. Physical Therapy Korea. 2009;16(1):61-9.

29. Vilca LW, Vallejos M. Construction of the risk of addiction to social networks scale(Cr. ARS). Computers in Human Behavior. 2015 Jul 1;48:190-8. https://doi.org/10.1016/j.chb.2015.01.049

30. Ghaem Panah E. The Study of the Relationship between Addiction to Social Networks and Online Games with Academic Performance in Hamadan University of Medical Sciences Students. Hamadan University of Medical Sciences; 2018.

31. Demetrovics Z, Urbán R, Nagygyörgy K, Farkas J, Griffiths MD, Pápay O, et al. The development of the problematic online gaming questionnaire (POGQ). PloS one. 2012 May 10;7(5):e36417.

32. Kuss DJ. Internet gaming addiction: current perspectives. Psychology research and behavior management. 2013;6:125. https://doi. org/10.2147/PRBM.S39476 PMID:24255603

33. Amini S, Nazari AM, Moradi A, Farzad V. Youth Online Gaming Addiction: The Role Of Self Esteem, Anxiety And Depression.

34. Van Rooij AJ, Schoenmakers TM, Vermulst AA, Van Den Eijnden RJ, Van De Mheen D. 


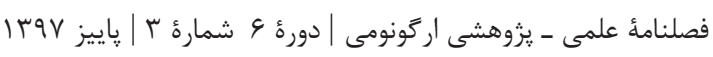

Online video game addiction: identification of addicted adolescent gamers. addiction. 2011 Jan;106(1):205-12.

35. Grüsser SM, Thalemann R, Griffiths MD. Excessive computer game playing: evidence for addiction and aggression?. CyberPsychology \& Behavior. 2006 Apr 1;10(2):290-2. https://doi. org $/ 10.1089 / \mathrm{cpb} .2006 .9956$

36. Andreassen CS. Online social network site addiction: A comprehensive review. Current Addiction Reports. 2015 Jun 1;2(2):175-84.

37. Al-Dubai SA, Ganasegeran K, Al-Shagga MA, Yadav H, Arokiasamy JT. Adverse health effects and unhealthy behaviors among medical students using Facebook. The scientific world journal. 2013;2013.

38. Asante KO, Nyarko J. The physical and behavioural consequences of Facebook use among university students. Mediterranean Journal of Social Sciences. 2014 Dec 10;5(27 P2):774.

39. Deogade SC, Saxena S, Mishra P. Adverse health effects and unhealthy behaviors among dental undergraduates surfing social networking sites. Industrial psychiatry journal. $2017 \mathrm{Jul} ; 26(2): 207$.

40. Choo H, Gentile D, Sim T, Li DD, Khoo A, Liau A. Pathological video-gaming among Singaporean youth. Ann Acad Med Singapore 2010;39:822-9.
41. Weiser EB. Gender differences in Internet use patterns and Internet application preferences: A two-sample comparison. Cyberpsychology and behavior. 2000 Apr 1;3(2):167-78. https://doi. org/10.1089/109493100316012

42. Ko CH, Yen JY, Chen CC, Chen SH, Yen CF. Gender differences and related factors affecting online gaming addiction among Taiwanese adolescents. The Journal of nervous and mental disease. 2005 Apr 1;193(4):273-7. https://doi. org/10.1097/01.nmd.0000158373.85150.57

43. Wijnhoven HA, de Vet HC, Picavet HS. Explaining sex differences in chronic musculoskeletal pain in a general population. Pain. 2006 Sep 1;124(1-2):158-66.

44. Picavet HS, Schouten JS. Musculoskeletal pain in the Netherlands: prevalences, consequences and risk groups, the DMC3-study. Pain. 2003 Mar 1;102(1-2):167-78.

45. DiFiori JP, Puffer JC, Mandelbaum BR, Mar S. Factors associated with wrist pain in the young gymnast. The American journal of sports medicine. 1996 Jan;24(1):9-14. https://doi. org $/ 10.1177 / 036354659602400103$

46. Subbarao JV, Klopfstein J, Turpin R. Prevalence and impact of wrist and shoulder pain in patients with spinal cord injury. The journal of spinal cord medicine. 1995 Jan 1;18(1):9-13. 
